\title{
ON 2-CLOSED ABELIAN PERMUTATION GROUPS
}

\author{
DMITRY CHURIKOV AND ILIA PONOMARENKO
}

\begin{abstract}
A permutation group $G \leq \operatorname{Sym}(\Omega)$ is said to be 2 -closed if no group $H$ such that $G<H \leq \operatorname{Sym}(\Omega)$ has the same orbits on $\Omega \times \Omega$ as $G$. A simple and efficient inductive criterion for the 2-closedness is established for abelian permutation groups with cyclic transitive constituents.
\end{abstract}

\section{INTRODUCTION}

The concept of $m$-closed permutation groups, $m \in \mathbb{N}$, was introduced by $\mathrm{H}$. Wielandt [10] in the framework of the method of invariant relations, developed him to study group actions. He proved that the $m$-closed groups are exactly the automorphism groups of the relational structures formed by families of $m$-ary relations on the same set. The 1-closed groups are just the direct product of symmetric groups. In the present paper, we are interested in the 2-closed groups.

Let $\Omega$ be a finite set and $G \leq \operatorname{Sym}(\Omega)$. Denote by $\operatorname{Orb}_{2}(G)$ the set of all orbits of the induced action of $G$ on $\Omega \times \Omega$. The 2-closure of the permutation group $G$ is defined to be the largest subgroup $\bar{G}=G^{(2)}$ in $\operatorname{Sym}(\Omega)$ that have the same 2-orbits as $G$, i.e.,

$$
\operatorname{Orb}_{2}(G)=\operatorname{Orb}_{2}(\bar{G}) \text {. }
$$

The group $G$ is said to be 2-closed if $G=\bar{G}$. The set $\operatorname{Orb}_{2}(G)$ forms a relational structure on $\Omega$, which is called a coherent configuration [1]. Thus the 2-closed groups are the automorphism groups of coherent configurations. This fact makes the concept of 2-closed groups especially important for algebraic combinatorics [5].

Based on the definition alone, it is difficult to determine whether a given permutation group $G$ is 2-closed. In some special cases (mostly for transitive groups the degree of which is the product of small number of primes), good criteria for the 2-closedness are obtained via the full classification of permutation groups of given degree, see, e.g., [3]. To the best of our knowledge, efficient algorithms recognizing 2-closed groups are known only for a few cases, e.g., for odd order groups 4 and for supersolvable groups $[8]$.

The present paper is motivated by the lack of good criteria for the 2-closedness even for abelian groups. A criterion found in [7, Theorem 6.1] does not seem quite satisfactory, because requires for a group $G$ to inspect all permutations in the direct product of the constituents $G^{\Delta}, \Delta \in \operatorname{Orb}(G)$. Our first result reduces the 2-closedness question to the case of $p$-groups, and generalize a Wielandt observation

The work is supported by Mathematical Center in Akademgorodok, the agreement with Ministry of Science and High Education of the Russian Federation number 075-15-2019-1613 and partially supported by the Russian Foundation for Basic Research (Grant 18-01-00752). 
that the classes of $p$-groups and abelian groups are invariant with respect to taking the 2-closure.

Theorem 1.1. Let $G$ be a nilpotent permutation group. Then $\bar{G}$ is nilpotent. Moreover,

$$
\bar{G}=\prod_{P \in \operatorname{Syl}(G)} \bar{P}
$$

Corollary 1.2. A nilpotent permutation group $G$ is 2-closed if and only if every Sylow subgroup of $G$ is 2-closed.

Probably, the first attempt to classify the 2-closed abelian groups was made by A. Zelikovskii in [11, Corollary 5]11 However, the characterization appears to be wrong and infinitely many counterexamples were found in [6]. To explain the gap in Zelikovskii's argument, let $G \leq \operatorname{Sym}(\Omega)$. One can associate with $G$ a permutation group on $\Omega$, defined as follows:

$$
\operatorname{Zel}(G)=\prod_{\Delta} \bigcap_{\Delta^{\prime} \neq \Delta} G_{\Delta^{\prime}}^{\Delta}
$$

where $\Delta$ and $\Delta^{\prime}$ run over the orbits of $G$, and $G_{\Delta^{\prime}}^{\Delta}=\left(G_{\Delta^{\prime}}\right)^{\Delta}$ is the restriction to $\Delta$ of the pointwise stabilizer of $\Delta^{\prime}$ in $G$. In this notation, the necessary and sufficient condition claimed by Zelikovskii for an abelian group $G$ to be 2-closed is that

$$
\operatorname{Zel}(G)^{\Delta}=\left(G_{\Omega \backslash \Delta}\right)^{\Delta} \quad \text { for all } \Delta \in \operatorname{Orb}(G) \text {. }
$$

It is not hard to see that this condition is equivalent to the inclusion $\operatorname{Zel}(G) \leq G$. Essentially, the only gap in Zelikovskii's argument consists in the wrong statement that if $G$ is 2-closed, then so is the permutation group induced by the action of $G$ on the orbits of $\operatorname{Zel}(G)$. In this way we arrive at the second result of the present paper.

Theorem 1.3. Let $G$ be an abelian permutation group and $Z=\operatorname{Zel}(G)$. Then $G$ is 2-closed if and only if $Z \leq G$ and $G^{\operatorname{Orb}(Z)}$ is 2-closed.

The "only if" part of Theorem 1.3 can be illustrated by the two following examples in which we construct non-2-closed groups $G$ and $H$, respectively. In the first example, $\operatorname{Zel}(G) \not \leq G$, whereas in the second one, $\operatorname{Zel}(H) \leq H$ but $H^{\operatorname{Orb}(\operatorname{Zel}(H))}$ is not 2-closed.

Example 1. Let $p$ be a prime, and let $G \leq \operatorname{Sym}(3 p)$ be an elementary abelian group of order $p^{2}$. The action of $G$ is chosen so that (a) there are exactly three $G$-orbits, each of size $p$, and (b) for any two points $\alpha$ and $\beta$ belonging to different $G$-orbits, the stabilizers $G_{\alpha}$ and $G_{\beta}$ are different subgroups of $G$ of order $p$ (if $\alpha$ and $\beta$ belong to the same orbit, then, of course, $\left.G_{\alpha}=G_{\beta}\right)$. From Wielandt's dissection theorem [10. Theorem 6.5], it follows that the group $\bar{G}$ equals the direct product of its transitive constituents; since $\bar{G}$ is abelian and $G \leq \bar{G}$, we conclude that $|\bar{G}|=p^{3}$. Thus, $G$ is not 2 -closed and $\bar{G}=\operatorname{Zel}(G)$.

Example 2. Let $\Omega_{1}$ and $\Omega_{2}$ be disjoint sets, and let $G_{1} \leq \operatorname{Sym}\left(\Omega_{1}\right)$ and $G_{2} \leq$ $\operatorname{Sym}\left(\Omega_{2}\right)$ be two copies of the permutation group $G$ from Example 1. For any two permutations $g_{1} \in G_{1}$ and $g_{2} \in G_{2}$, corresponding to the same element of $G$, we

\footnotetext{
${ }^{1}$ The cited statement was formulated for arbitrary intransitive groups with 2-closed transitive constituents.
} 
define a permutation $g \in \operatorname{Sym}\left(\Omega_{1} \cup \Omega_{2}\right)$ such that $g^{\Omega_{i}}=g_{i}, i=1,2$. Let $H$ be the group of all these permutations $g$; this group is isomorphic to $G$ as abstract group and have the same point stabilizers as $G$ (again as abstract groups). Then $H_{\Delta^{\prime}}^{\Delta}=1$ for any two orbits $\Delta$ and $\Delta^{\prime}$ corresponding to the same orbit of $G$. It follows that $H$ is not 2-closed (see Lemma 5.1) and $\operatorname{Zel}(H)=1$.

Theorem 1.3 reduces the question on the 2-closedness of abelian group $G$ to the case when the group $\operatorname{Zel}(G)$ is trivial. This case can really happen even if $G$ is nontrivial, see Example 2. In a large class of abelian permutation groups, one can continue the reduction by "removing" unessential orbits defined as follows. An orbit $\Delta$ of a group $G \leq \operatorname{Sym}(\Omega)$ is said to be unessential if $G$ is 2-closed if and only if $G^{\Omega \backslash \Delta}$ is 2-closed.

Theorem 1.4. Let $G$ be an intransitive group and $p$ a prime. Assume that every transitive constituent of $G$ is a cyclic p-group. Then $\operatorname{Zel}(G)$ is trivial only if every orbit of $G$ is unessential.

Combining the obtained results we arrive at the following simple and efficient inductive criterion of the 2-closedness for an (abelian) permutation group $G$ with cyclic transitive constituents. First, there is nothing to do if $G$ is transitive, because in this case $G$ is 2-closed by [10, Example 5.13.]. Second, Theorem 1.1 reduces the problem to the case when $G$ is a $p$-group. At this point a further reduction is needed. Namely, depending on whether the group $Z=\operatorname{Zel}(G)$ is trivial, we continue with

$$
G^{\operatorname{Orb}(Z)} \text { or } G^{\Omega \backslash \Delta} \text {, }
$$

where $\Delta$ is an arbitrarily chosen orbit of $G$. The correctness is provided by Theorems 1.3 and 1.4. respectively. Each of these two reductions decreases the degree of a group and hence the test is finished after at most $|\Omega|$ reductions.

The proofs of Theorems 1.1. 1.3. and 1.4 are presented in Sections 3.4 and 5 respectively. The relevant notation and definitions are collected in Section 2

The authors thank S. Skresanov and A. Vasil'ev for their suggestions for improving the text.

\section{Permutation groups}

Throughout the paper, $\Omega$ is a finite set. For a permutation group $G \leq \operatorname{Sym}(\Omega)$, we use the notation

$$
\operatorname{Orb}(G)=\operatorname{Orb}(G, \Omega)=\left\{\alpha^{G}: \alpha \in \Omega\right\}
$$

for the set of $G$-orbits $\alpha^{G}=\left\{\alpha^{g}: g \in G\right\}$. A set $\Delta \subseteq \Omega$ is said to be $G$-invariant if $\Delta^{g}=\Delta$ for all $g \in G$. In this case, we denote by $G^{\Delta}$ the permutation group on $\Delta$, induced by the natural action of $G$ on $\Delta$; it consists of the permutations $g^{\Delta} \in \operatorname{Sym}(\Delta), g \in G$, taking $\delta$ to $\delta^{g}, \delta \in \Delta$.

In general, when $G$ acts on a set $\Delta$ (which is not necessarily a subset of $\Omega$ ), we denote by $G^{\Delta}$ the subgroup of $\operatorname{Sym}(\Delta)$, induced by the corresponding action.

For an arbitrary $\Delta \subseteq \Omega$, we denote by $G_{\Delta}$ and $G_{\{\Delta\}}$ the pointwise and setwise stabilizer of $\Delta$ in $G$, respectively. Thus, if $\Delta$ is $G$-invariant, then $G_{\Delta}$ is the kernel of the restriction homomorphism $G \rightarrow G^{\Delta}$, whereas $G_{\{\Delta\}}=G$. 
The groups $G, H \leq \operatorname{Sym}(\Delta)$ are said to be 2-equivalent if $\operatorname{Orb}_{2}(G)=\mathrm{Orb}_{2}(H)$. Formula (11) implies that $G$ and $\bar{G}$ are 2-equivalent. In fact, the 2-closure $\bar{G}$ is the largest group in the class of all groups 2-equivalent to $G$.

All other undefined terms and notation are standard and can be found in 2 .

\section{Proof of Theorem 1.1}

Let $G \leq \operatorname{Sym}(\Omega)$ be a nilpotent group of degree $n=|\Omega|$. For a prime $p \mid n$, the largest $p$-power divisor of $n$ is denoted by $n_{p}$; if $\pi$ is a set of prime divisors of $n$, then we put $n_{\pi}:=\prod_{p \in \pi} n_{p}$. The set of all prime divisors of the order of $G$ is denoted by $\pi(G)$.

Lemma 3.1. Assume that $G$ is transitive and $H$ a Hall subgroup of $G$. Then

(1) the size of every $H$-orbit is equal to $n_{\pi}$, where $\pi=\pi(H)$,

(2) $G$ acts on $\operatorname{Orb}(H)$; moreover, the kernel of this action is equal to $H$.

Proof. The nilpotency of $G$ implies that $H \unlhd G$. Therefore, $G$ permutes the orbits of $H$ and hence acts on $\Delta=\operatorname{Orb}(H)$. Denote by $L$ the kernel of this action.

The transitivity of $G$ and normality of $H$ imply that all $H$-orbits have the same size $m$. In particular, $m$ divides $n$. Since $n$ divides $|H|$, this implies that $m$ also divides $|H|$. Taking into account that $\operatorname{GCD}(n,|H|)$ divides $n_{\pi}$, we conclude that

$m$ divides $n_{\pi}$.

Next, the group $G^{\Delta} \leq \operatorname{Sym}(\Delta)$ is transitive. Consequently, $|\Delta|$ divides $\left|G^{\Delta}\right|$. However the numbers $\left|G^{\Delta}\right|$ and $|H|$ are coprime, because $H \leq L$ and $H$ is a Hall subgroup of $G$. Since also $|\Delta|$ divides $n$, this implies that $|\Delta|$ divides $n_{\pi^{\prime}}$, where $\pi^{\prime}$ is the set of prime divisors of $n$, not belonging to $\pi$. Together with (3) this shows that

$$
n=m \cdot|\Delta| \leq n_{\pi} \cdot n_{\pi^{\prime}}=n,
$$

whence $m=n_{\pi}$. This proves statement (1).

To prove statement (2), assume on the contrary that $H<L$. Then there exists an element $g \in L$ of prime order $q \notin \pi$. Denote by $\Gamma$ the orbit of $L$ such that $g^{\Gamma} \neq \mathrm{id}_{\Gamma}$. Then the Sylow $q$-subgroup $Q$ of $L^{\Gamma}$ is nontrivial. Since $L^{\Gamma}$ is transitive and nilpotent, we can apply statement (1) to $G=L^{\Gamma}$ and $H=Q$. Then

$$
|\Gamma|=|\Gamma|_{q} \cdot|\operatorname{Orb}(Q)| .
$$

On the other hand, we can apply statement (1) to $G=L^{\Gamma}$ and $H=H^{\Gamma}$. Since $\Gamma$ is also an orbit of $H$, this statement implies that

$$
|\Gamma|=|\Gamma|_{\pi} \cdot\left|\operatorname{Orb}\left(H^{\Gamma}\right)\right|=|\Gamma|_{\pi} .
$$

Thus, $|\Gamma|_{q} \cdot|\operatorname{Orb}(Q)|=|\Gamma|_{\pi}$. However, the numbers $|\Gamma|_{q}$ and $|\Gamma|_{\pi}$ are relatively prime. Therefore $|\Gamma|_{q}=1$ and hence $|\Gamma|=|\operatorname{Orb}(Q)|$. It follows that $Q=1$, a contradiction.

Lemma 3.2. Theorem 1.1 holds if $G$ is transitive.

Proof. If $G$ is a $p$-group, then $\bar{G}$ is a $p$-group [10, Exercise 5.28] and the required statement is true. Thus we may assume that $G=P \times H$, where $P$ and $H$ are the Sylow $p$-subgroup and Hall subgroup of $G$, respectively. Let $\Delta \in \operatorname{Orb}(P)$ and $\Gamma \in \operatorname{Orb}(H)$. By Lemma 3.1(1), we have

$$
|\Delta|=n_{p} \quad \text { and } \quad|\Gamma|=n_{p^{\prime}},
$$


where $p^{\prime}=\pi(H)$. Further, $\Delta$ and $\Gamma$ are blocks of the transitive group $G$. Therefore, the intersection $\Delta \cap \Gamma$ is either empty or is a block the size of which divides both $|\Delta|$ and $|\Gamma|$. Thus, $|\Delta \cap \Gamma| \leq 1$.

Each point $\alpha \in \Omega$ lies in exactly one $P$-orbit, say $\Delta_{\alpha}$, and in exactly one $H$-orbit, say $\Gamma_{\alpha}$. By the above, $\left|\Delta_{\alpha} \cap \Gamma_{\alpha}\right|=1$. Consequently, the mapping

$$
\rho: \Omega \rightarrow \operatorname{Orb}(P) \times \operatorname{Orb}(H), \alpha \mapsto\left(\Delta_{\alpha}, \Gamma_{\alpha}\right)
$$

is a bijection. Denote by $P^{\prime}$ and $H^{\prime}$ the permutation groups induced by the actions of $G$ on $\operatorname{Orb}(H)$ and on $\operatorname{Orb}(P)$, respectively. By Lemma 3.1(2), we have

$$
P^{\rho}=P^{\prime} \times 1 \text { and } H^{\rho}=1 \times H^{\prime} .
$$

Thus the group $G$ can be identified with the direct product $P^{\prime} \times H^{\prime}$ acting on $\operatorname{Orb}(H) \times \operatorname{Orb}(P)$. From [4, Proposition 3.1(2)], it follows that

$$
\left(P^{\prime} \times H^{\prime}\right)^{(2)}=\left(P^{\prime}\right)^{(2)} \times\left(H^{\prime}\right)^{(2)},
$$

which completes the proof by induction on the number $|\pi(G)|$ with taking into account that $\pi(G)=\pi(P) \cup \pi(H)$.

Let us return to the general case. Any transitive constituent $H$ of $G$ is a homomorphic image of $G$ which is nilpotent. Therefore, $H$ is nilpotent and $\pi(H) \subseteq \pi(G)$. By Lemma 3.2, the group $\bar{H}$ is nilpotent and also $\pi(H)=\pi(\bar{H})$. In view of [4, Proposition 3.1(1)], this implies that the group

$$
\bar{G} \leq\left(\prod_{H} H\right)^{(2)}=\prod_{H} \bar{H}
$$

is a subgroup of the direct product of nilpotent groups. Consequently, $\bar{G}$ is nilpotent and $\pi(G)=\pi(\bar{G})$. Thus it remains to verify that if $P$ and $Q$ are Sylow $p$-subgroups of $G$ and $\bar{G}$, respectively, then $\bar{P}=Q$. We need two auxiliary lemmas.

Lemma 3.3. Let $P$ and $Q$ as above. Then $\bar{P} \leq Q$. Moreover,

$$
\operatorname{Orb}(P)=\operatorname{Orb}(Q) \text {. }
$$

Proof. The first part of the statement follows from the monotonicity of the 2-closure operator; in particular, $P \leq \bar{P} \leq Q$. Thus, to prove the second part, it suffices to verify that each $P$-orbit $\Delta$ is a $Q$-orbit. Denote by $\Gamma$ the $G$-orbit containing $\Delta$. Then $\Gamma$ is also a $\bar{G}$-orbit. It follows that there exists a $Q$-orbit $\Delta^{\prime}$ such that

$$
\Delta \subseteq \Delta^{\prime} \subseteq \Gamma
$$

The group $G^{\Gamma}$ and $\bar{G}^{\Gamma}$ are transitive and nilpotent. By Lemma 3.1(1), this implies that $|\Delta|=|\Gamma|_{p}=\left|\Delta^{\prime}\right|$. This shows that $\Delta=\Delta^{\prime}$, as required.

Lemma 3.4. Let $\Delta, \Gamma \in \operatorname{Orb}(P)$. Then $\left(G_{\{\Delta\}} \cap G_{\{\Gamma\}}\right)^{\Delta \cup \Gamma} \leq P^{\Delta \cup \Gamma}$.

Proof. The statement is trivial if $G=P$. Now let $G=P \times H$, where $H$ is a Hall subgroup of $G$. It follows that each $g \in G$ can be written as $g=x y$ with $x \in P$ and $y \in H$. Assume that

$$
g \in G_{\{\Delta\}} \cap G_{\{\Gamma\}},
$$

i.e., $g$ leaves $\Delta$ and $\Gamma$ fixed (as sets). Then the permutation $x^{-1} \in P$ leaves the sets $\Delta$ and $\Gamma$ fixed, because they are $P$-orbits. Thus, $\Delta^{y}=\Delta^{x^{-1} g}=\Delta^{g}=\Delta$ and similarly, $\Gamma^{y}=\Gamma$. 
We claim that

$$
y^{\Delta}=\mathrm{id}_{\Delta} \quad \text { and } \quad y^{\Gamma}=\mathrm{id}_{\Gamma} .
$$

Let us prove the first equality; the second one is proved analogously. The permutation $y^{\Delta}$ belongs to the centralizer $Z$ of transitive group $P^{\Delta}$ in $\operatorname{Sym}(\Delta)$. According to [9, Exercise 4.5'], the group $Z$ is semiregular. In particular, $|Z|$ divides $|\Delta|$ which is a $p$-power. Therefore, $Z$ is a $p$-group. Consequently, the order of $y^{\Delta}$ is a $p$-power and hence $y^{\Delta} \in P^{\Delta}$. Taking into account that $P^{\Delta} \cap H^{\Delta}=1$, we conclude that the first equality in (5) holds.

Using equalities (5), we have

$$
g^{\Delta \cup \Gamma}=(x y)^{\Delta \cup \Gamma}=x^{\Delta \cup \Gamma} y^{\Delta \cup \Gamma}=x^{\Delta \cup \Gamma} \in P^{\Delta \cup \Gamma},
$$

as required.

To complete the proof of the theorem, we note that $Q$ is 2-closed, for otherwise $\bar{Q}$ is a $p$-subgroup of $\bar{G}$, containing the Sylow $p$-subgroup $Q$. Thus it suffices to verify that given $\alpha, \beta \in \Omega$ and $\bar{g} \in Q$, there exists $h \in P$ such that

$$
(\alpha, \beta)^{\bar{g}}=(\alpha, \beta)^{h} \text {. }
$$

The 2-equivalence of $G$ and $\bar{G}$ implies that $(\alpha, \beta)^{\bar{g}}=(\alpha, \beta)^{g}$ for some $g \in G$. Denote by $\Delta$ and $\Gamma$ the $Q$-orbits containing the points $\alpha$ and $\beta$, respectively. Then obviously, $\alpha^{g}=\alpha^{\bar{g}}$ belongs to $\Delta$ and $\beta^{g}=\beta^{\bar{g}}$ belongs to $\Gamma$. Therefore,

$$
\alpha, \alpha^{g} \in \Delta \text { and } \beta, \beta^{g} \in \Gamma \text {. }
$$

In view of equality (4), $\Delta$ and $\Gamma$ are also $P$-orbits. Since the group $G$ permute the $P$-orbits, it follows that $g \in G_{\{\Delta\}} \cap G_{\{\Gamma\}}$. By Lemma 3.4(1), there exists $h \in P$ such that $h^{\Delta \cup \Gamma}=g^{\Delta \cup \Gamma}$. Thus,

$$
(\alpha, \beta)^{\bar{g}}=(\alpha, \beta)^{g}=(\alpha, \beta)^{h},
$$

as required. Theorem 1.1 is completely proved.

Proof of Corollary 1.2. Let $G$ be a nilpotent permutation group. Assume that $G$ is 2 -closed. Then $G=\bar{G}$ and hence $\operatorname{Syl}(G)=\operatorname{Syl}(\bar{G})$. It remains to note that by Theorem 1.1. any Sylow subgroup of $\bar{G}$ is 2-closed. Conversely, assume that $P=\bar{P}$ for each $P \in \operatorname{Syl}(G)$. Then again by Theorem 1.1 we have

$$
\bar{G}=\prod_{P \in \operatorname{Syl}(G)} \bar{P}=\prod_{P \in \operatorname{Syl}(G)} P=G,
$$

i.e., $G$ is 2 -closed.

\section{Proof of Theorem 1.3}

It is well known that a transitive abelian group is regular [9, Proposition 4.4]. Therefore every abelian permutation group $G$ is quasiregular, i.e., every transitive constituent of $G$ is regular. Thus Theorem 1.3 is an immediate consequence of the following theorem.

Theorem 4.1. Let $G$ be a quasiregular permutation group and $Z=\operatorname{Zel}(G)$. Then $G$ is 2-closed if and only if $Z \leq G$ and $G^{\operatorname{Orb}(Z)}$ is 2-closed.

Proof. Let $\Delta$ be a $G$-orbit. Then the group $G^{\Delta}$ is regular and hence 2-closed. Since $\bar{G}$ is contained in the direct product of the 2-closures of the groups $G^{\Delta}$, this shows that $\bar{G}$ is quasiregular. In what follows, we assume that $G \leq \operatorname{Sym}(\Omega)$. 
Lemma 4.2. $Z \leq \bar{G}$, and also $Z^{\Delta} \unlhd \bar{G}^{\Delta}$ for all $\Delta \in \operatorname{Orb}(\bar{G})$.

Proof. The group $Z$ is generated by the permutations $z$ satisfying the following condition: there exists $\Delta \in \operatorname{Orb}(Z)$ such that

$$
z^{\Delta} \in Z^{\Delta} \text { and } z^{\Omega \backslash \Delta}=\mathrm{id}_{\Omega \backslash \Delta} .
$$

Thus to prove the inclusion $Z \leq \bar{G}$, it suffices to verify that each such $z$ belongs to $\bar{G}$, or equivalently that $s^{z}=s$ for every $s \in \operatorname{Orb}_{2}(G)$.

Denote by $\Delta^{\prime}$ and $\Delta^{\prime \prime}$ the $G$-orbits such that $s \subseteq \Delta^{\prime} \times \Delta^{\prime \prime}$. If $\Delta^{\prime} \neq \Delta \neq \Delta^{\prime \prime}$, then $s^{z}=s$ by the second equality in (6), whereas if $\Delta=\Delta^{\prime}=\Delta^{\prime \prime}$, then $s \in \operatorname{Orb}_{2}\left(G^{\Delta}\right)$ and again $s^{z}=s$, because $Z^{\Delta} \leq G^{\Delta}$. Thus without loss of generality, we may assume that $\Delta=\Delta^{\prime \prime} \neq \Delta^{\prime}$. Then by the definition of $Z$ (see formula (2)), there exists $g \in G_{\Delta^{\prime}}$ such that

$$
z^{\Delta \cup \Delta^{\prime}}=g^{\Delta \cup \Delta^{\prime}}
$$

Thus, $s^{z}=s^{g}=s$, as required.

Let us prove that $Z^{\Delta} \unlhd \bar{G}^{\Delta}$. We have $G_{\Delta^{\prime}} \unlhd G$ for every $\Delta^{\prime} \in \operatorname{Orb}(G)$. Therefore, $G_{\Delta^{\prime}}^{\Delta} \unlhd G^{\Delta}$. Consequently, the intersection of all $G_{\Delta^{\prime}}^{\Delta}$ taken over all $\Delta^{\prime} \neq \Delta$ is also normal in $G^{\Delta}$. Since this intersection coincides with $Z^{\Delta}$ and $G^{\Delta}=\bar{G}^{\Delta}$, we are done.

From Lemma 4.2 it follows that for every $\Delta \in \operatorname{Orb}(\bar{G})$, the group $\bar{G}^{\Delta}$ acts on the set $\operatorname{Orb}\left(Z^{\Delta}\right)$. Therefore, $\bar{G}$ acts on the union $\operatorname{Orb}(Z)$ of all $\operatorname{Orb}\left(Z^{\Delta}\right)$. Denote by $\bar{\rho}$ the corresponding epimorphism from $\bar{G}$ to $\bar{G}^{\operatorname{Orb}(Z)}$. Then obviously $Z$ is a subgroup of $L:=\operatorname{ker}(\bar{\rho})$. Moreover, if $\Delta$ is a $\bar{G}$-orbit, then

$$
L^{\Delta}=Z^{\Delta},
$$

because $L^{\Delta}$ and $Z^{\Delta}$ are 1-equivalent subgroups of the regular group $\bar{G}^{\Delta}$ (recall that the group $\bar{G}$ is quasiregular). Since $L$ is contained in the direct product of the $Z^{\Delta}$, the definition of $Z$ implies that $L=Z$. This proves the following statement.

Lemma 4.3. $\operatorname{ker}(\bar{\rho})=Z$.

The epimorphism $\bar{\rho}$ induces the action of $G \leq \bar{G}$ on the set $\operatorname{Orb}(Z)$; denote by $\rho$ the corresponding epimorphism from $G$ to $G^{\operatorname{Orb}(Z)}$. It should be noted that while the group $Z$ is not, in general, a subgroup of $G$, the permutation group $G^{\operatorname{Orb}(Z)}$ is well defined.

Lemma 4.4. $\operatorname{im}(\bar{\rho})=\overline{G^{\mathrm{Orb}(Z)}}$.

Proof. The groups $G^{\operatorname{Orb}(Z)}$ and $\bar{G}^{\mathrm{Orb}(Z)}$ are 2-equivalent [8, Lemma 2.1(1)]. Therefore,

$$
\operatorname{im}(\bar{\rho})=\bar{G}^{\operatorname{Orb}(Z)} \leq \overline{G^{\operatorname{Orb}(Z)}} .
$$

Conversely, we need to verify that for every $\bar{g} \in \overline{G^{\operatorname{Orb}(Z)}}$, there exists $g \in \bar{G}$ such that

$$
\bar{\rho}(g)=\bar{g} .
$$

Let $\Delta \in \operatorname{Orb}(\bar{G})$. The quasiregularity of $\bar{G}$ implies that the group $\bar{G}^{\Delta}=G^{\Delta}$ is regular. Since also $Z^{\Delta} \unlhd \bar{G}^{\Delta}$ (Lemma 4.2), the group $\left(\bar{G}^{\Delta}\right)^{\operatorname{Orb}\left(Z^{\Delta}\right)}$ is also regular and hence 2-closed. It follows that

$$
\left(\overline{G^{\operatorname{Orb}(Z)}}\right)^{\bar{\Delta}} \leq \overline{\left(G^{\Delta}\right)^{\operatorname{Orb}\left(Z^{\Delta}\right)}}=\overline{\left(\bar{G}^{\Delta}\right)^{\operatorname{Orb}\left(Z^{\Delta}\right)}}=\left(\bar{G}^{\Delta}\right)^{\operatorname{Orb}\left(Z^{\Delta}\right)},
$$


where $\bar{\Delta}$ is the $\overline{G^{\mathrm{Orb}(Z)}}$-orbit the points of which are the $Z$-orbits contained in $\Delta$. Thus for every $\Delta \in \operatorname{Orb}(\bar{G})$, there exists a permutation $g_{\Delta} \in \bar{G}^{\Delta}$ such that

$$
\bar{\rho}_{\Delta}\left(g_{\Delta}\right)=\bar{g}^{\bar{\Delta}}
$$

where $\bar{\rho}_{\Delta}$ is the epimorphism from $\bar{G}^{\Delta}$ to $\left(\bar{G}^{\Delta}\right)^{\operatorname{Orb}\left(Z^{\Delta}\right)}$, induced by $\bar{\rho}$. Now if the product $g$ of all the $g_{\Delta}$ lies in $G$, then

$$
\bar{\rho}(g)=\bar{\rho}\left(\prod_{\Delta} g_{\Delta}\right)=\prod_{\Delta} \bar{\rho}_{\Delta}\left(g_{\Delta}\right)=\prod_{\bar{\Delta}} \bar{g}^{\bar{\Delta}}=\bar{g},
$$

which proves equality (7).

It remains to verify that $g \in \bar{G}$. To this end, let $s \in \mathrm{Orb}_{2}(G)$. Then $s \in \mathrm{Orb}_{2}(\bar{G})$ and hence $s \subseteq \Delta \times \Gamma$ for some $\Delta, \Gamma \in \operatorname{Orb}(\bar{G})$. Now if $\Delta=\Gamma$, then $s^{g}=s^{g_{\Delta}}=s$, because $g_{\Delta} \in \bar{G}^{\Delta}$. Assume that $\Delta \neq \Gamma$. Then by Lemma 4.3 and the definition of $Z$, we have $Z^{\Delta} \times Z^{\Gamma} \subseteq \bar{G}^{\Delta \cup \Gamma}$. It follows that

$$
(\alpha, \beta) \in s \quad \Leftrightarrow \quad \bar{\alpha} \times \bar{\beta} \subseteq s,
$$

where $\bar{\alpha}=\alpha^{Z}$ and $\bar{\beta}=\beta^{Z}$. Furthermore, the set $\bar{s}=\{(\bar{\alpha}, \bar{\beta}):(\alpha, \beta) \in s\}$ is a 2 -orbit of the group $\bar{G}^{\operatorname{Orb}(Z)}$ and hence $\bar{s}^{\bar{g}}=\bar{s}$. Thus,

$$
s^{g}=\left(\bigcup_{(\bar{\alpha}, \bar{\beta}) \in \bar{s}} \bar{\alpha} \times \bar{\beta}\right)^{g}=\bigcup_{(\bar{\alpha}, \bar{\beta}) \in \bar{s}} \bar{\alpha}^{\bar{g}} \times \overline{\beta^{g}}=\bigcup_{(\bar{\alpha}, \bar{\beta}) \in \bar{s}^{\bar{g}}} \bar{\alpha} \times \bar{\beta}=\bigcup_{(\bar{\alpha}, \bar{\beta}) \in \bar{s}} \bar{\alpha} \times \bar{\beta}=s
$$

as required.

To prove the "only if" part, assume that the group $G$ is 2-closed. Then by Lemma 4.2. we have $Z \leq \bar{G}=G$, whereas by Lemma 4.4, we have

$$
G^{\mathrm{Orb}(Z)}=\bar{G}^{\mathrm{Orb}(Z)}=\operatorname{im}(\bar{\rho})=\overline{G^{\mathrm{Orb}(Z)}},
$$

i.e., the group $G^{\operatorname{Orb}(Z)}$ is 2-closed, as required.

To prove the "if" part, assume that $Z \leq G$ and the group $G^{\operatorname{Orb}(Z)}$ is 2-closed. By Lemma 4.3, the first condition implies that

$$
Z \leq \operatorname{ker}(\rho) \leq \operatorname{ker}(\bar{\rho})=Z,
$$

in particular, $\operatorname{ker}(\rho)=\operatorname{ker}(\bar{\rho})$. Furthermore, by Lemma 4.4 the second condition implies that $\overline{G^{\operatorname{Orb}(Z)}}=G^{\operatorname{Orb}(Z)}$ and hence

$$
\operatorname{im}(\rho)=G^{\operatorname{Orb}(Z)}=\overline{G^{\operatorname{Orb}(Z)}}=\operatorname{im}(\bar{\rho}) .
$$

Thus,

$$
|G|=|\operatorname{ker}(\rho)| \cdot|\operatorname{im}(\rho)|=|\operatorname{ker}(\bar{\rho})| \cdot|\operatorname{im}(\bar{\rho})|=|\bar{G}| .
$$

Since $G \leq \bar{G}$, this means that $G=\bar{G}$, i.e., $G$ is 2-closed.

\section{Proof of Theorem 1.4}

We begin with a sufficient condition for an orbit of quasiregular permutation group to be unessential. The proof is based on a special result from theory of coherent configurations [1].

Lemma 5.1. Let $G$ be a quasiregular permutation group and $\Delta \in \operatorname{Orb}(G)$. Assume that $G_{\Delta^{\prime}}^{\Delta}=1$ for some $G$-orbit $\Delta^{\prime} \neq \Delta$. Then the orbit $\Delta$ is unessential. 
Proof. The quasiregularity of $G$ implies that $G_{\delta^{\prime}}=G_{\Delta^{\prime}}$ for each $\delta^{\prime} \in \Delta^{\prime}$. It follows that if $\delta, \lambda \in \Delta$, then

$$
\left(\delta^{\prime}, \delta\right) \in\left(\delta^{\prime}, \lambda\right)^{G} \Rightarrow \delta=\lambda .
$$

Indeed, if $\left(\delta^{\prime}, \delta\right)=\left(\delta^{\prime}, \lambda\right)^{g}$ for some $g \in G$, then $g \in G_{\delta^{\prime}}=G_{\Delta^{\prime}}$. Since $G_{\Delta^{\prime}}^{\Delta}=1$, this implies that $\delta=\lambda^{g}=\lambda$.

Denote by $\Omega$ the point set of $G$ and put $S=\operatorname{Orb}_{2}(G)$. Then the pair $\mathcal{X}=(\Omega, S)$ is a coherent configuration and $\bar{G}=\operatorname{Aut}(\mathcal{X})$ is the automorphism group of $\mathcal{X}$, see [1. Formula (8) implies that the condition (3.3.14) from [1] is satisfied for the coherent configuration $\mathcal{X}$ and the set $\Delta$ equal to

$$
\Omega^{\prime}:=\Omega \backslash \Delta .
$$

By [1, Lemma 3.3.20(1)], the restriction homomorphism $\operatorname{Aut}(\mathcal{X}) \rightarrow \operatorname{Aut}\left(\mathcal{X}_{\Omega^{\prime}}\right)$ is an isomorphism; in particular,

$$
\bar{G}^{\Omega^{\prime}}=\operatorname{Aut}(\mathcal{X})^{\Omega^{\prime}}=\operatorname{Aut}\left(\mathcal{X}_{\Omega^{\prime}}\right)=\overline{G^{\Omega^{\prime}}} .
$$

To prove that the orbit $\Delta$ is unessential, first assume that the group $G$ is 2-closed. Then formula (9) shows that the group $G^{\Omega^{\prime}}=\bar{G}^{\Omega^{\prime}}$ is also 2-closed. Conversely, assume that $G^{\Omega^{\prime}}$ is 2-closed. Then $G^{\Omega^{\prime}}=\overline{G^{\Omega^{\prime}}}$. Consequently, the restriction homomorphism $G \rightarrow G^{\Omega^{\prime}}$ is surjective. Since $G \leq \bar{G}=\operatorname{Aut}(\mathcal{X})$, the homomorphism is also injective. Thus, $G=\bar{G}$, i.e., $G$ is 2-closed.

Turn to the proof of Theorem 1.4. Assume that the group $\operatorname{Zel}(G)$ is trivial. Then for each $\Delta \in \operatorname{Orb}(G)$, we have

$$
\bigcap_{\Delta^{\prime} \neq \Delta} G_{\Delta^{\prime}}^{\Delta}=1 .
$$

On the other hand, by the theorem hypothesis, $G_{\Delta^{\prime}}^{\Delta} \leq G^{\Delta}$ is a cyclic p-group for all $G$-orbits $\Delta^{\prime}$. Thus, $G_{\Delta^{\prime}}^{\Delta}=1$ for at least one $\Delta^{\prime}$. By Lemma 5.1, this implies that the orbit $\Delta$ is unessential.

\section{REFERENCES}

[1] G. Chen and I. Ponomarenko, Coherent Configurations, Central China Normal University Press, Wuhan (2019).

[2] J. D. Dixon and B. Mortimer, Permutation Groups, Graduate Texts in Mathematics, 163, Springer (1996).

[3] E. Dobson and I. Kovács, Automorphism groups of Cayley digraphs of $\mathbb{Z}_{p}^{3}$, Electron. J. Combin., 16, no. 1, Research Paper 149 (2009).

[4] S. Evdokimov and I. Ponomarenko, Two-closure of odd permutation group in polynomial time, Discrete Math., 235, No. 1-3, 221-232 (2001).

[5] S. Evdokimov and I. Ponomarenko, Permutation group approach to association schemes, European J. Combin., 30, No. 6, 1456-1476 (2009).

[6] M. Grech and A. Kisielewicz, 2-Closed Abelian Permutation Groups, Electron. Notes Discrete Math., 68, 83-88 (2018).

[7] M. Grech and A. Kisielewicz, Abelian permutation groups with graphical representations, arXiv:1910.11816 [math.CO], 1-20 (2019).

[8] I. Ponomarenko and A. Vasil'ev, Two-closure of supersolvable permutation group in polynomial time, Computational Complexity, 29, 5 (2020); https://doi.org/10.1007/s00037-02000195-7.

[9] H. Wielandt, Finite Permutation Groups, Academic press, New York - London (1964).

[10] H. Wielandt, Permutation Groups Through Invariant Relations and Invariant Functions, Lect. Notes Dept. Math. Ohio St. Univ., Columbus (1969). 
[11] A. Zelikovskii, The König problem for abelian permutation groups [in Russian], Vestsī Akad. Navuk BSSR Ser. Fīz.-Mat. Navuk, No. 5, 34-39 (1989).

DMITRY CHURIKOV

Sobolev Institute of Mathematics,

PR. Koptyuga, 4,

630090, Novosibirsk, Russia

Novosibirsk State University,

Ul. Pirogova, 2,

630090, Novosibirsk, Russia

churikovdv@gmail.com

Ilia PonOmarenko

Steklov Institute of Mathematics at St. Petersburg,

FONTANKA, 27,

191023, St. Petersburg, Russia

Sobolev Institute of Mathematics,

PR. Koptyuga, 4,

630090, Novosibirsk, Russia

inp@pdmi.ras.ru 